\title{
Nitrogen- and Oxygen-Containing Three-Dimensional Hierarchical Porous Graphitic Carbon for Advanced Supercapacitor
}

\author{
Yunyan Zhao, Honghu Wang, Jing Liu, Jinghao Liu, Guicun Li *, Hongrui Peng, Kezheng Chen \\ and Zhonghua Zhang * \\ College of Materials Science and Engineering, Qingdao University of Science and Technology, \\ Qingdao 266042, China; zhaoyy@qust.edu.cn (Y.Z.); tzswhh@126.com (H.W.); jingliu@qust.edu.cn (J.L.); \\ liujh@glabat.com (J.L.); penghongrui@qust.edu.cn (H.P.); kchen@qust.edu.cn (K.C.) \\ * Correspondence: guicunli@qust.edu.cn (G.L.); zhangzh@qust.edu.cn (Z.Z.); Tel.: +86-532-84022900 (G.L.); \\ Fax: +86-532-84022900 (G.L.)
}

Received: 5 July 2020; Accepted: 3 August 2020; Published: 6 August 2020

\begin{abstract}
Three-dimensional hierarchical porous graphitic carbon (HPGC) were synthesized via one-step carbonization-activation and a catalytic strategy. The method can not only improve the graphitization degree of carbon materials, but also offer plentiful interfaces for charge accumulation and short paths for ion/electron transport. Polypyrrole, potassium hydroxide, and nickel acetate were used as the carbon precursors, activating agent, and catalyst, respectively. The retraction and dissolution of $\mathrm{Ni}$ caused the change of pore size in the material and led to the interconnected micro/nano holes. Nickel acetate played a significant role in enhancing the electrical conductivity, introducing pseudocapacitance, and promoting ion diffusion. In the supercapacitor, HPGC electrode exhibited a remarkable specific capacitance of $336.3 \mathrm{~F} \mathrm{~g}^{-1}$ under $0.5 \mathrm{~A} \mathrm{~g}^{-1}$ current density and showed high rate capability, even with large current densities applied (up to $50 \mathrm{~A} \mathrm{~g}^{-1}$ ). Moreover, HPGC showed optimal cycling stability with $97.4 \%$ capacitance retention followed by 3000 charge-discharge cycles. The excellent electrochemical performances coupled with a facile large-scale synthesis procedure make HPGC a promising alternative for supercapacitors.
\end{abstract}

Keywords: hierarchical porous structures; porous graphitic carbon; polypyrrole; supercapacitors; Ni catalyst

\section{Introduction}

As a novel type of energy-storage device, supercapacitors have recently received considerable attention since they are essential components of high-rate electric devices in hybrid vehicles [1-4]. Activated carbons with a large specific surface area (SSA) over $2000 \mathrm{~m}^{2} \mathrm{~g}^{-1}$, one type of electrode materials for supercapacitors, have achieved commercial success in supercapacitor devices due to their low cost [5-7]. The mechanism of energy storage is based on the accumulation of charge in the electric double layer formed at the electrode/electrolyte interface $[8,9]$. It is reported that some nitrogen- and oxygen-containing functional groups play important roles in enhancing the electrochemical performance of the carbon-based materials due to the introduction of pseudocapacitance and improving electrolyte wettability [10-12]. Compared with heteroatoms doping in carbon-based materials, using heteroatoms-containing polymers as precursors is an easier way to obtain nitrogenand oxygen-containing carbon [13]. Heteroatoms-containing polymers mainly contain biomass, natural polymers and synthetic polymers [14]. Among them, synthetic polymer precursors are very popular because the surface/interface properties and chemical structure of the precursors can be tailored and 
designed. Polypyrrole (PPy) has great potential for preparing carbon-based materials due to the simple synthetic method, low cost, large specific surface area, good processability, and chemical stability.

However, there still exist several disadvantages for carbon-based materials, such as the sluggish ion diffusion and low electrical conductivity [15-17]. Sluggish ion diffusion may cause capacity loss and low power density during the fast charging/discharging process [18]. In recent years, some studies have illustrated that hierarchical porous carbon (HPC) has a high specific surface area and interconnected pores structure to guarantee the rapid electrolyte ions transfer within the electrode materials $[19,20]$. Typically, HPC can be obtained by either physical activation with different oxidizing atmospheres or chemical activation with $\mathrm{KOH}, \mathrm{H}_{3} \mathrm{PO}_{4}$, or $\mathrm{ZnCl}_{2}$ [20]. According to the literature, oxygen- and nitrogen-enriched porous carbon materials showed high specific capacitance with excellent cycling stability [20-22].

Nevertheless, the low electron conductivity of the above carbon materials limits their rate capability and energy efficiency. The electrical conductivity of carbon-based materials can be effectively improved by graphitization [23]. Generally, high-temperature or/and high-pressure treatment of carbon precursor and catalytic graphitization are major methods to improve the degree of graphization [24]. Catalytic graphitization is a better option, to avoid the vast collapse of pore structure [25]. Even so, a high graphitization degree may lead to an undeveloped pore structure and the reduction of the specific surface area, and further lead to the sluggish ion diffusion [26]. However, our research found that the catalyst caused the collapse of macropores $(>50 \mathrm{~nm})$ and micropores $(<2 \mathrm{~nm})$, but created more mesopores (from 2 to $50 \mathrm{~nm}$ ) and the interconnection of pores.

Herein, we report a facile large-scale synthesis method of activated carbon, which can not only improve the graphitization degree, but also offer plentiful interfaces for charge accumulation and short paths for ion/electron transport. Novel functionalized hierarchical porous graphitic carbon (HPGC) with interconnected micro/nano holes was synthesized via simultaneous activation of $\mathrm{KOH}$ and catalysis of $\mathrm{Ni}(\mathrm{Ac})_{2} \cdot 4 \mathrm{H}_{2} \mathrm{O}$ using PPy as the precursor. The presence of nickel catalyst not only increases the graphitization degree of the material, but also creates more interconnected pores. Hence, our HPGC displayed excellent electrochemical performance and a high lifetime cycling stability.

\section{Experimental}

\subsection{Synthesis of Hierarchical Porous Graphitic Carbon (HPGC) Materials}

The PPy was obtained in a chemical polymerization step using a templateless procedure $[27,28]$. In a typical synthesis, $2 \mathrm{~mL}$ of pyrrole monomer was dissolved in $290 \mathrm{~mL}$ of $0.1 \mathrm{~mol} \mathrm{~L}^{-1} \mathrm{HCl}$ solution under ultrasonication for $15 \mathrm{~min}$, and then $50 \mathrm{~mL}$ of $\left(\mathrm{NH}_{4}\right)_{2} \mathrm{~S}_{2} \mathrm{O}_{8}(6.8 \mathrm{~g})$ aqueous solution was added to the above solution. The molar ratio of pyrrole monomer, doping agent $(\mathrm{HCl})$, and oxidizing agent (ammonium persulfate) was 1:1:1. The mixture was magnetically stirred for $5 \mathrm{~min}$. After that, the polymerization reaction occurred at low temperature $\left(0-5{ }^{\circ} \mathrm{C}\right)$ for $24 \mathrm{~h}$ without any disturbance. The formed PPy products were filtered and washed with $3 \mathrm{wt} . \%$ ammonia water solution, deionized water, and ethanol several times until the filtrate became neutral. The as-prepared PPy products $(2 \mathrm{~g})$ and $\mathrm{Ni}(\mathrm{Ac})_{2} \cdot 4 \mathrm{H}_{2} \mathrm{O}(1.44 \mathrm{~g})$ were dispersed in $50 \mathrm{~mL}$ of $25 \mathrm{v} / \mathrm{v} \%$ ethanol/deionized water solution by stirring at room temperature for $10 \mathrm{~h}$. Subsequently, $6 \mathrm{~g}$ of $\mathrm{KOH}$ was added into the mixture under stirring. Then, the solvent was removed via vacuum evaporation at $50{ }^{\circ} \mathrm{C}$. The obtained powder was carbonized in a tubular furnace under nitrogen atmosphere at $700{ }^{\circ} \mathrm{C}$ for $2 \mathrm{~h}$ with a heating rate of $3{ }^{\circ} \mathrm{C} \mathrm{min}^{-1}$. After carbonization, the inorganic impurities were removed with enough deionized water to obtain hierarchical porous graphitic carbon@ nickel (HPGC-Ni) composite (Figure 1). Finally, HPGC-Ni composite was etched in $1 \mathrm{~mol} \mathrm{~L}^{-1}$ of $\mathrm{HCl}$ solution at $80^{\circ} \mathrm{C}$ for $24 \mathrm{~h}$ to remove $\mathrm{Ni}$ and impurities (residual $\mathrm{KOH}$ ), washed with deionized water, and then dried at $80{ }^{\circ} \mathrm{C}$ in an oven for $12 \mathrm{~h}$ to obtain HPGC. For comparison, HPC was obtained under the same conditions without $\mathrm{Ni}$ $(\mathrm{Ac})_{2} \cdot 4 \mathrm{H}_{2} \mathrm{O}$. Analytical-grade pyrrole was purchased from Aldrich (Shanghai, China). Other chemical reagents were purchased from Sanhe (Yantai, China) and used as received. 


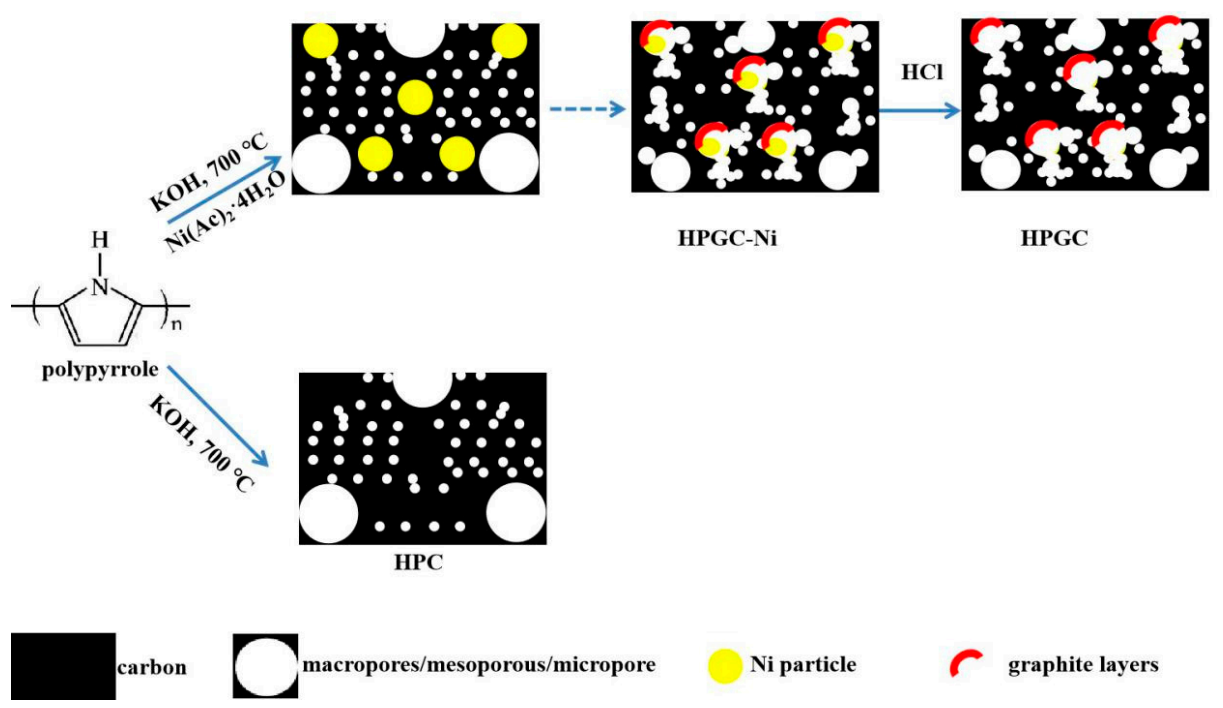

Figure 1. Schematic showing the preparation of hierarchical porous carbon (HPC) and hierarchical porous graphitic carbon (HPGC).

\subsection{Materials Characterization}

The morphology of the samples was investigated by transmission electron microscopy (TEM, FEI Tecnai G20, (FEI Ltd., Hillsboro, OR, USA)) and field-emission scanning electron microscopy (SEM, JSM6700F, JEOL Ltd., Tokyo, Japan). Energy-dispersive spectrometer (EDS) mapping images were also carried out on the JSM6700F electron microscope (JEOL Ltd., Tokyo, Japan) operated at $20 \mathrm{kV}$. The high-resolution transmission electron microscopy (HRTEM) images were carried out on a JEOL JEM-2010 electron microscope (JEOL Ltd., Tokyo, Japan) operated at $200 \mathrm{kV}$. X-ray diffraction (XRD) analysis was conducted with a Rigaku D-max-X- $\gamma$ A diffractometer (Rigaku Ltd., Tokyo, Japan) and $\mathrm{Cu} \mathrm{K} \alpha$ radiation $(\lambda=1.54178 \AA)$. Nitrogen adsorption-desorption isotherms were measured on a micrometrics ASAP 2020 analyzer (Micromeritics Instrument corp., Atlanta, GA, USA) at $-196{ }^{\circ} \mathrm{C}$. Before measurements, the samples were degassed at $150{ }^{\circ} \mathrm{C}$ under vacuum for $4 \mathrm{~h}$. A nitrogen adsorption/desorption test (Micromeritics ASAP2020) was used for obtaining the overall specific surface area by the Brunauer-Emmett-Teller (BET) theory. The total pore volume, calculated from the adsorbed amount at a relative pressure of 0.99, was determined by the Barrett-Joyner-Halenda (BJH) method. X-ray photoelectron spectroscopy (XPS) experiments were performed at a Perkin-Elmer PHI 550 spectrometer (Perkin-Elmer corp., Waltham, MA, USA). The XPS spectra were recorded using monochromatized radiation energy of $1486.6 \mathrm{eV}$. Deconvolution of XPS peaks was performed by a CasaXPS software (version 2.3.13, Casa Software Ltd., Beijing, China). Fourier transform infrared spectroscopy (FTIR) was obtained with a CARY500UV-VIS-NI spectrometer (Cary Ltd., Palo Alto, CA, USA) in a range from $4000 \mathrm{~cm}^{-1}$ to $400 \mathrm{~cm}^{-1}$.

\subsection{Electrochemical Measurements}

The electrochemical performance of the samples was measured via a three-electrode system in a $1 \mathrm{~mol} \mathrm{~L}^{-1}$ of $\mathrm{H}_{2} \mathrm{SO}_{4}$ electrolyte solution $[17,29]$. The $\mathrm{Pt}$ foil and saturated calomel electrode (SCE) were taken as counter electrode and reference electrode, respectively. The reference voltage was 0.245 V. All electrochemical tests were assessed on an Autolab Potentiostat/Galvanostat, PGSTAT302N. The working electrodes were prepared by mixing activated carbon $(75 \mathrm{wt} \%)$, acetylene black $(20 \mathrm{wt} \%)$, and polytetraflfluoroethylene binder $(5 \mathrm{wt} \%)$ in ethanol solvent [30]. The slurry was rolled on a stainless steel $(1 \times 1 \mathrm{~cm})$ current collector and dried overnight at $80^{\circ} \mathrm{C}$. The as-prepared electrodes were pressed under a pressure of $10 \mathrm{MPa}$ for $60 \mathrm{~s}$ and then dried at $80^{\circ} \mathrm{C}$ for $24 \mathrm{~h}$. Cyclic voltammetry (CV) was performed in a potential range from $-0.2 \mathrm{~V}$ to $0.8 \mathrm{~V}$ by varying the scan rate from 5 to $100 \mathrm{mV} \mathrm{s}^{-1}$. Galvanostatic charge-discharge measurements were performed at various current densities 
of $0.5-50 \mathrm{~A} \mathrm{~g}^{-1}$. For the Nyquist plots were obtained in the frequency range of $10 \mathrm{KHz}$ to $10 \mathrm{MHz}$ at open circuit potential. Polytetraflfluoroethylene was purchased from Aldrich (Shanghai, China). Other chemical reagents were purchased from Sanhe (Yantai, China) and used as received.

\section{Results and Discussion}

The uniform distribution of Ni element in HPGC-Ni is critical to the electrical conductivity of HPGC. The position of Ni catalyst in HPGC-Ni determines the distribution of conducting graphitized carbon and quaternary N in HPGC. SEM images of HPGC-Ni and the corresponding EDS mapping images are displayed in Figure 2 to investigate the distribution of Ni. HPGC-Ni was formed through the process of carbonization, activation, and catalysis. SEM image shows that the HPGC-Ni sample exhibits an alveolate-like porous structure with a pore diameter of about $1 \mu \mathrm{m}$. The porous structure was generated due to the etch of $\mathrm{KOH}$ activation reagent through a redox reaction [26]. Besides, there are many Ni particles with a diameter of about $200 \mathrm{~nm}$ can be seen in the carbon matrix. Ni particles were formed by the carbothermal reduction of nickel acetate. The C, N, and O elements in EDS mapping images come from the precursor PPy. The corresponding EDS mapping images display the distribution of $\mathrm{Ni}$ element is consistent with that of $\mathrm{C}, \mathrm{N}$, and $\mathrm{O}$ elements, indicating that Ni nanoparticles are uniformly encapsulated into the carbon frameworks.

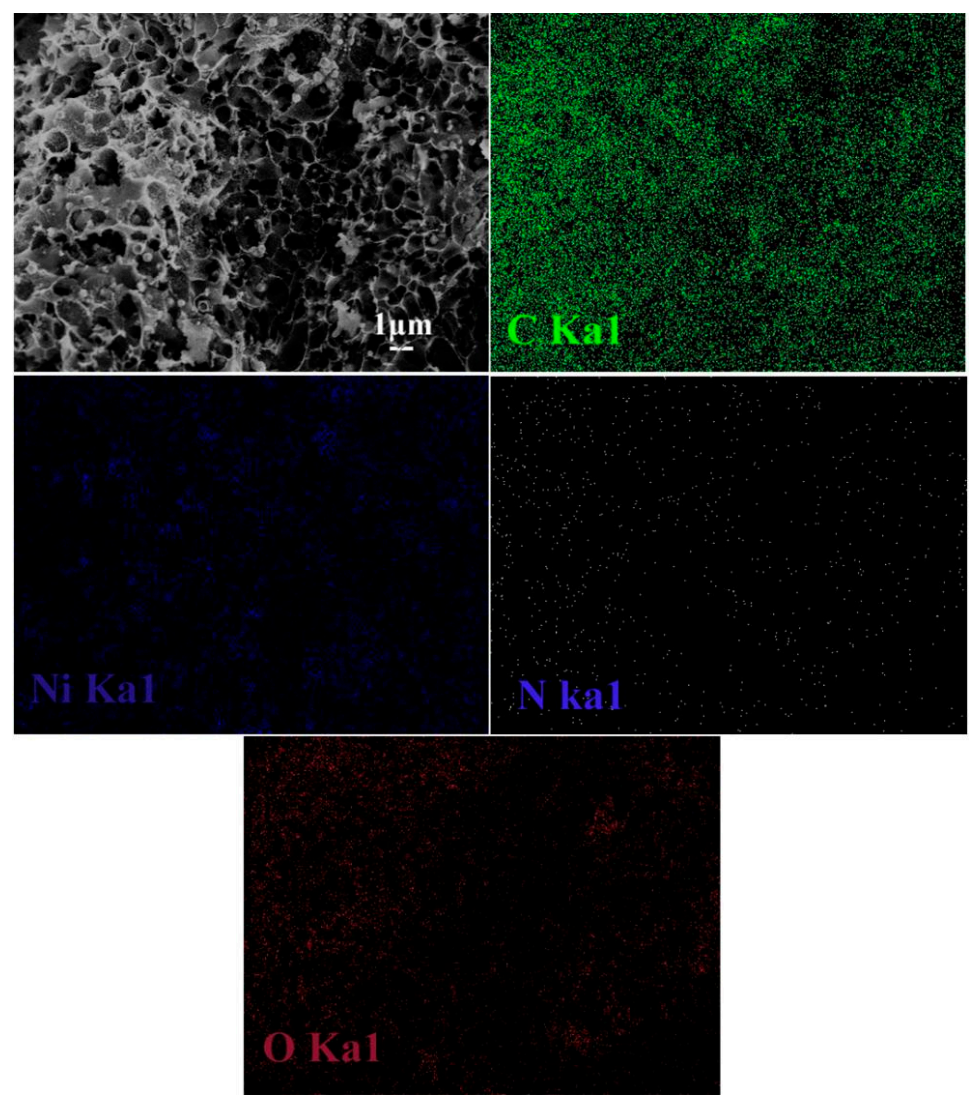

Figure 2. Scanning electron microscopy (SEM) image of HPGC-Ni and the corresponding energy-dispersive spectrometer (EDS) mapping images.

The existential form of nickel element in HPGC-Ni, and the graphitization degree of HPGC caused by nickel element were investigated by XRD patterns in Figure 3. As shown in Figure 3a, the diffraction peaks of HPGC-Ni located at $44.2^{\circ}, 51.5^{\circ}$, and $76.1^{\circ}$ can be indexed to the (111), (200), and (220) crystal planes of metallic Ni (Joint Committee on Powder Diffraction Standards, JCPDS card, no. 65-0380), respectively [31]. Nickel existed as an elementary substance, which agrees with the result from SEM images of HPGC-Ni. The diffraction peaks of carbon cannot be found due to the high crystallinity 
of nickel. XRD patterns of HPGC shows that the diffraction of peaks of Ni disappeared after the etching of $\mathrm{HCl}$ solution, indicating that the Ni nanoparticles were completely removed (Figure 3b). The disappearance of Ni nanoparticles is validated by the following SEM image of HPGC. Both HPGC and HPC show one broad peak at $24.7^{\circ}$ due to amorphous carbon structures. Two peaks of HPGC at $25.6^{\circ}$ and $42.7^{\circ}$ correspond to (002) and (101) planes of graphitized carbon, respectively [2]. According to Bragg's equation, the $d_{002}$ value for HPGC material is calculated to be $0.345 \mathrm{~nm}$, which is smaller than that of HPC $(0.36 \mathrm{~nm})$. The narrower $\mathrm{d}_{002}$ value implies higher crystallinity and degree of graphitization, which can also be demonstrated by HRTEM images. As shown in Figure 4e, no distinct lattice distance can be found in the HRTEM image, suggesting the amorphous state of the HPC. However, the HRTEM image of HPGC in Figure $4 \mathrm{f}$ shows the distinct crystal lattice distance with a large interlayer space of $0.34 \mathrm{~nm}$, in line with the interplane distance of the (002) lattice of graphite layers [25]. The result indicates a highly graphitized structure of HPGC, owing to the catalytic action Ni nanoparticle [32]. The formation mechanism of graphitized structure is shown in Figure 1. At high temperature, the Ni metal crystals ripen and amorphous carbon is taken up by Ni. After that, the carbon film is dissolved and graphene layers nucleate and grow on the Ni surfaces. Finally, $\mathrm{Ni}$ retracts and graphitized carbon becomes visible due to the inherent instability of $\mathrm{Ni}$ lamella and high temperature. Increased electrical conductivity of HPGC caused by a high graphitization degree can contribute to high rate capability and energy efficiency.
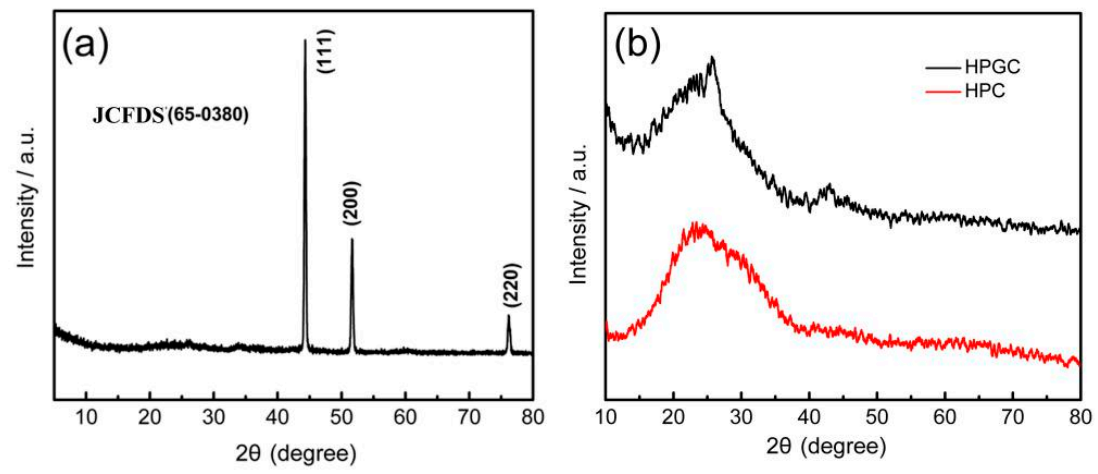

Figure 3. X-ray diffraction (XRD) patterns of HPGC-Ni (a), HPGC and HPC (b).

The presence of nickel catalyst not only increases graphitization degree of material, but also changes morphology of material and creates more interconnected pores. To observe the morphology of HPGC, representative SEM, TEM, and HRTEM images were recorded with HPC for comparison. The SEM image of HPGC in Figure $4 \mathrm{~b}$ exhibit a three-dimensional alveolate-like morphology with abundant open and interconnected pores. The disappearance of Ni particles in HPGC is in keeping with the result of the XRD pattern. The SEM image of HPC is similar to that of HPGC, but HPC has significantly fewer folds and holes. The formation of the pores is attributed to not only the etching effect of the $\mathrm{KOH}$ activation reagent, but also the retraction and disappearance of $\mathrm{Ni}$. A few macropores of 2-10 $\mu \mathrm{m}$ in HPC changes to abundant macropores of $<2 \mu \mathrm{m}$ in HPGC due to the presence of nickel catalyst. Large quantities of macroporous voids can provide ion-buffering reservoirs and minimize the diffusion distance to the interior surface during the charge and discharge process. Therefore, the abundant macropores in HPGC can bring about high power density and high-rate capacitive performance. TEM images in Figure 4c,d reveal that both HPC and HPGC samples has plenty of micropores, mesopores, and macropores. However, there are more interconnected micro/nano holes in HPGC, which offered vast active sites for electrochemical reactions, plentiful interfaces for charge accumulation, and short paths for ion/electron transport. 

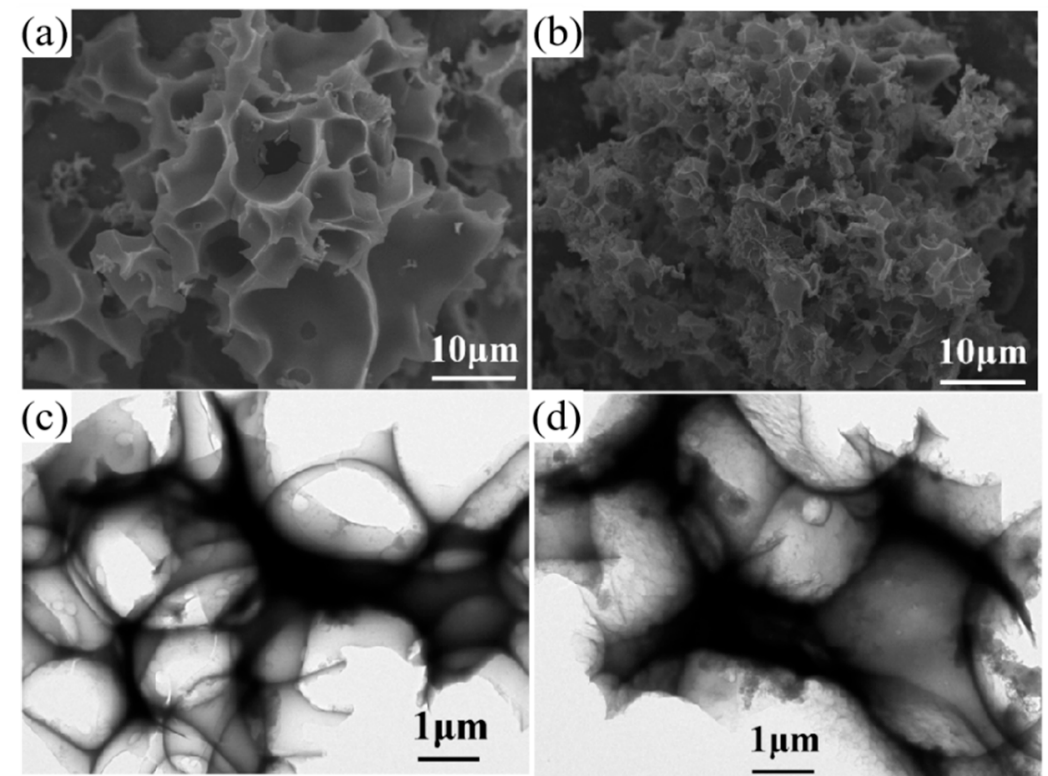

(d)

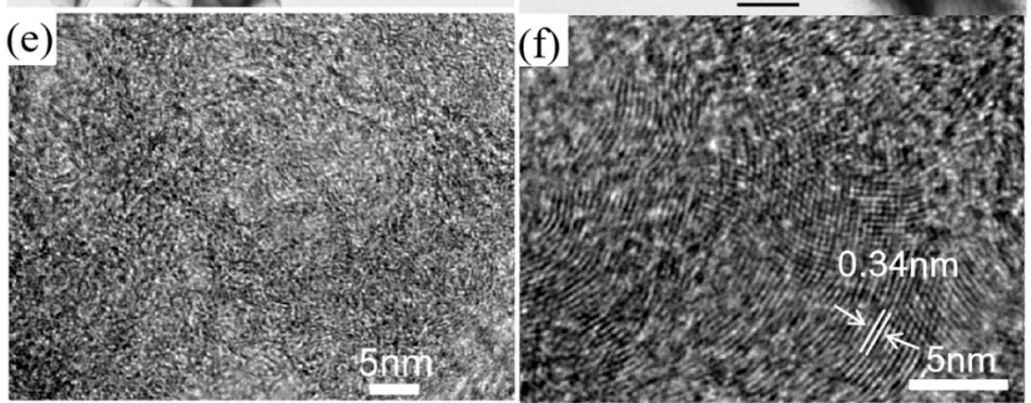

Figure 4. SEM (a,b), transmission electron microscopy (TEM) (c,d), and high-resolution TEM (HRTEM) $(\mathbf{e}, \mathbf{f})$ images of HPC $(\mathbf{a}, \mathbf{c}, \mathbf{e})$ and HPGC $(\mathbf{b}, \mathbf{d}, \mathbf{f})$ samples.

Pore size distribution and the specific surface area are important factors for the capacity of the electric double-layer capacitor. $\mathrm{N}_{2}$ adsorption-desorption isotherms were tested to further analyze the pore structures of HPC and HPGC (Figure 5a). Both HPC and HPGC present combined features of type-I isotherm and type-IV isotherm. A steep of nitrogen uptake at a low relative pressure indicates the formation of numerous micropores, while a hysteresis at a high relative pressure $(\mathrm{P} / \mathrm{P} 0=0.8-1.0)$ suggests a mesoporous material. The BET specific surface area of HPGC and HPC was calculated to be $2489.2 \mathrm{~m}^{2} \mathrm{~g}^{-1}$ and $3000.6 \mathrm{~m}^{2} \mathrm{~g}^{-1}$, respectively. The pore volume of HPGC and HPC calculated by the $\mathrm{BJH}$ method was $1.61 \mathrm{~cm}^{3} \mathrm{~g}^{-1}$ and $2.11 \mathrm{~cm}^{3} \mathrm{~g}^{-1}$, respectively. As shown, the larger specific surface area and pore volume of HPC is attributed to more micropores. However, the decrease of ion-transport resistance depends mostly on mesopores in porous carbons [21]. Figure $5 b$ shows the pore size distribution curves of HPC and HPGC samples. The similar pore size distribution and BET specific surface area are determined by $\mathrm{KOH}$ activation. Moreover, $\mathrm{HPC}$ displays a relatively narrow pore size distribution of 1-7 nm. However, the pore size of HPGC centers on 1-5 nm and $25-45 \mathrm{~nm}$. For HPGC, the retraction of $\mathrm{Ni}$ at high temperature may cause the change of pore size and lead to more mesopores of $25-45 \mathrm{~nm}$. Compared with pores of 1-5 nm, the mesopores of 25-45 nm are beneficial to ions transport. The nitrogen adsorption-desorption measurements join morphology proving the interconnection of micro/nano holes in HPGC. The structure of interconnection may be due to the retraction and dissolution of $\mathrm{Ni}$, which is beneficial for the charge storage and ion transfer (Figure 1). The graphitized hierarchical porous structure could facilitate fast electron and electrolyte ion transport during the charge and discharge process, thereby improving the capacitance at high current density. 


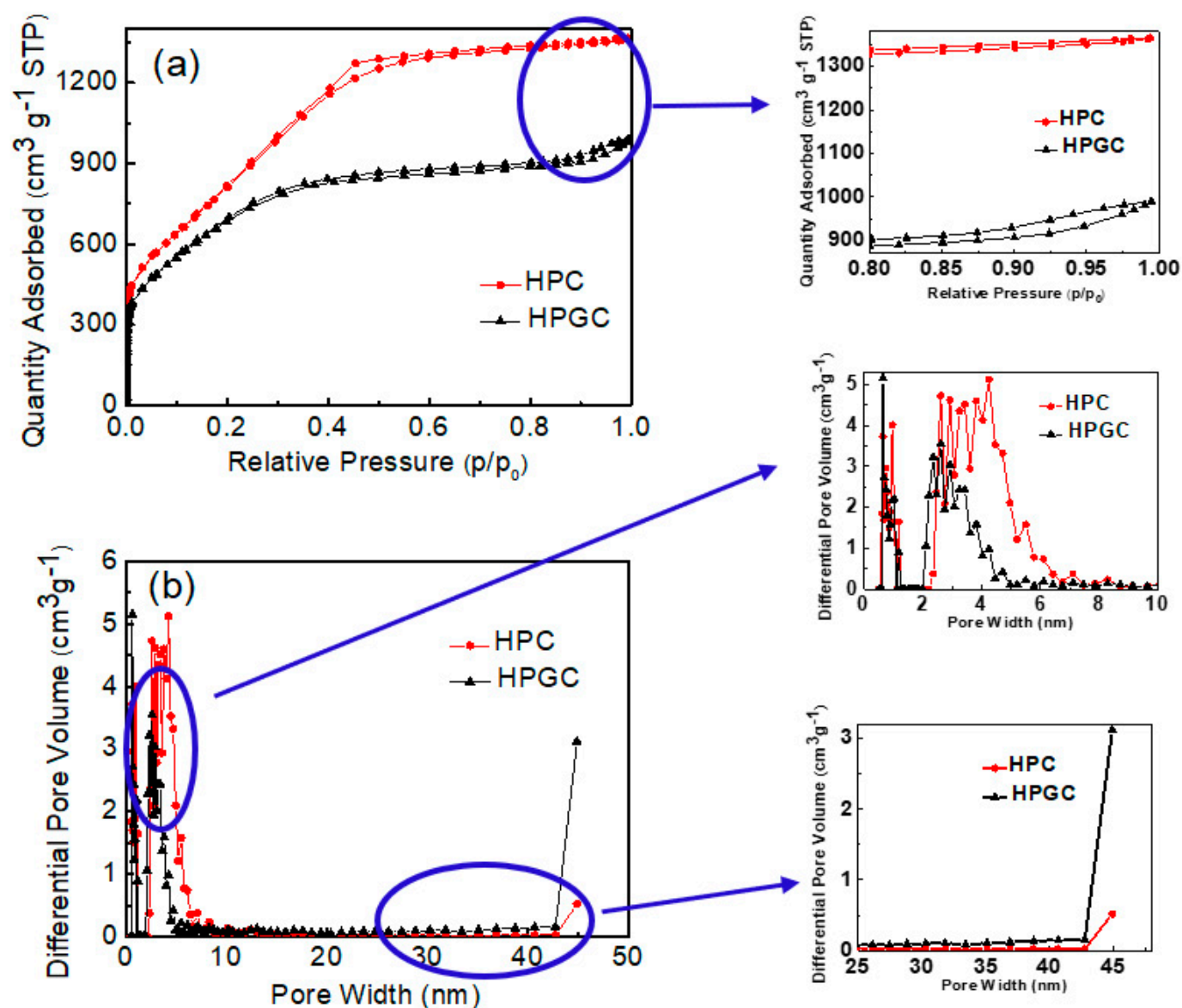

Figure 5. $\mathrm{N}_{2}$ adsorption-desorption isotherms (a) and pore size distribution curves (b) of HPC and HPGC samples (the inset figures show enlarged curves).

The capacitance of carbon materials can also be improved by heteroatoms. The chemical states and element content of samples were analyzed by the XPS survey spectra of HPGC and HPC (Figure 6). The atomic percentages of $\mathrm{C}, \mathrm{N}$ and $\mathrm{O}$ calculated from XPS spectra are summarized in Table 1. It can be seen that the O content increases from 5.91 at.\% for HPC to 9.11 at.\% for HPGC, and the percentage of $\mathrm{N}$ decreases from 7.04 at.\% for HPC to 3.52 at.\% for HPGC. N and C elements of two samples originate from precursor PPy. The FTIR spectrum of PPy (Figure 7) presents the characteristic peaks at 1560 (C-C stretching vibration), 1298 (C-N in-plane deformation), 1191 (C-C bending vibration), 1041 (C-H in-plane deformation), 922 (C-H out-of-plane ring deformation), $789(\mathrm{C}-\mathrm{H})$ and $675 \mathrm{~cm}^{-1}$ (C-C out-of-plane ring deformation) [33]. The increase of the $\mathrm{O}$ content is attributed to the incorporation of oxygen in nickel acetate during the carbonization-activation process. HPGC and HPC two carbon materials have almost the similar total content of heteroatoms. The high-resolution $\mathrm{N} 1 \mathrm{~s}$ spectrum can be resolved into three individual peaks corresponding to pyridinic $\mathrm{N}(398.4 \pm 0.3 \mathrm{eV})$, pyrrolic $\mathrm{N}$ $(400.3 \pm 0.3 \mathrm{eV})$, and quaternary $\mathrm{N}(401.1 \pm 0.3 \mathrm{eV})$, respectively (Figure 6b,c) [16]. Compared with HPC, HPGC had higher quaternary N content which was 29.29 at.\% (Table 1). It can be concluded that the presence of $\mathrm{Ni}$ catalyst is beneficial to the formation of quaternary $\mathrm{N}$ during the carbonization process of the PPy precursor. Quaternary N possesses strong ability of giving electrons, which can effectively enhance the conductivity of the material and improve capacity retention at high rates. Furthermore, pyrrolic $\mathrm{N}$ and pyridinic $\mathrm{N}$ can produce additional pseudocapacitance, while quaternary $\mathrm{N}$ can enhance the ability of generating pseudocapacitance. The existence of surface oxygen functional groups is attributed to the incorporation of oxygen from potassium hydroxide and nickel acetate. For HPGC in Figure 6d, the high-resolution 

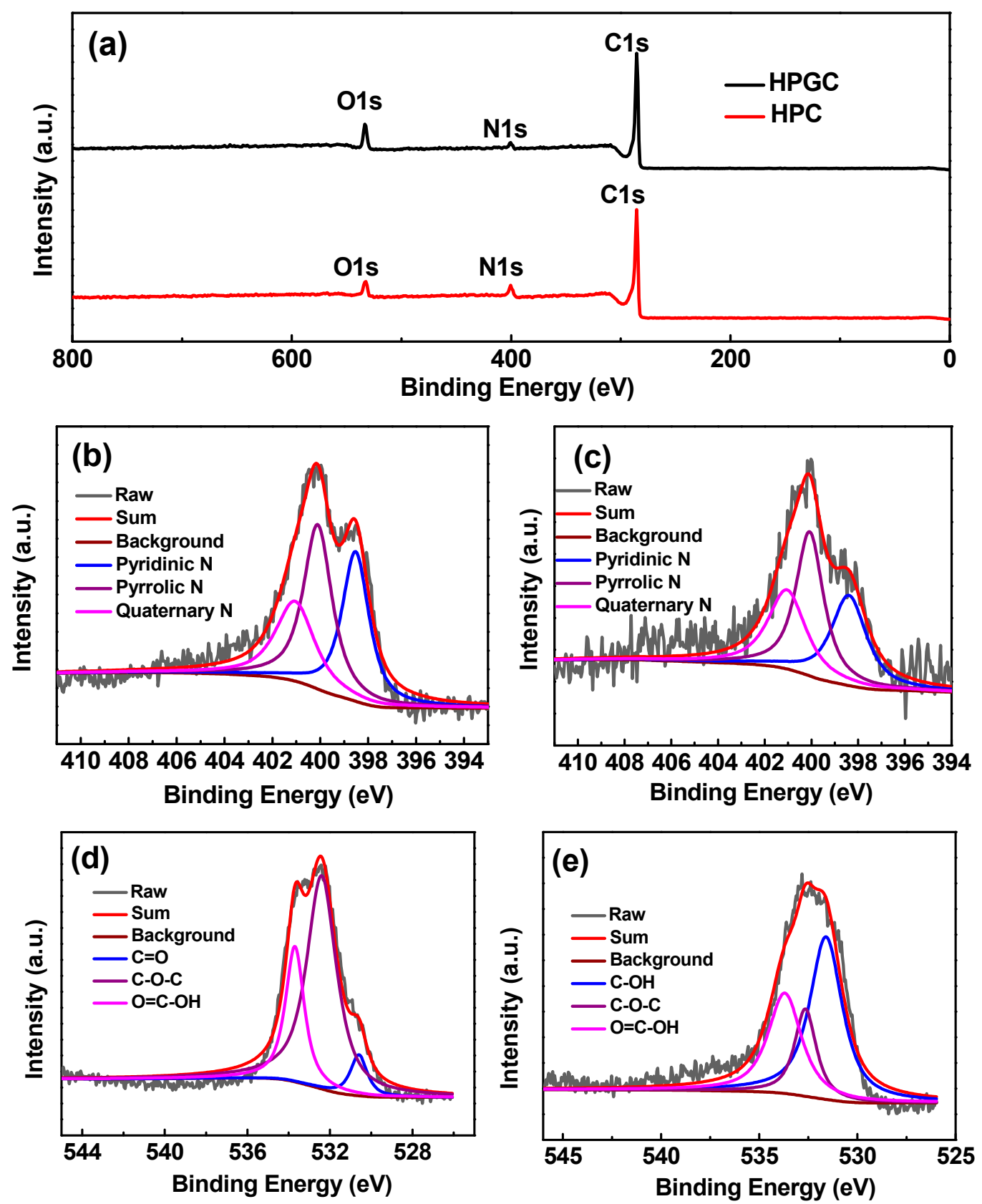

Figure 6. X-ray photoelectron spectroscopy (XPS) survey spectra (a) of the HPGC and HPC samples; High-resolution XPS spectra of N1s $(\mathbf{b}, \mathbf{c})$ and O1s $(\mathbf{d}, \mathbf{e})$ peaks of the HPGC sample $(\mathbf{b}, \mathbf{d})$ and the HPC sample (c,e).

Table 1. The C, N and O contents in the HPGC and HPC samples obtained from XPS spectra.

\begin{tabular}{|c|c|c|c|c|c|c|}
\hline \multirow{2}{*}{ Samples } & \multirow{2}{*}{$\mathrm{C}_{\text {XPS }}$ (at.\%) } & \multirow{2}{*}{$\mathrm{N}_{\text {XPS }}$ (at.\%) } & \multirow{2}{*}{$\mathrm{O}_{\mathrm{XPS}}$ (at.\%) } & \multicolumn{3}{|c|}{ Nitrogen Contents from XPS (at.\%) } \\
\hline & & & & Pyridinic N & Pyrrolic N & Quaternary N \\
\hline HPC & 87.05 & 7.04 & 5.91 & 34.37 & 39.48 & 26.15 \\
\hline HPGC & 87.37 & 3.52 & 9.11 & 31.48 & 39.23 & 29.29 \\
\hline
\end{tabular}




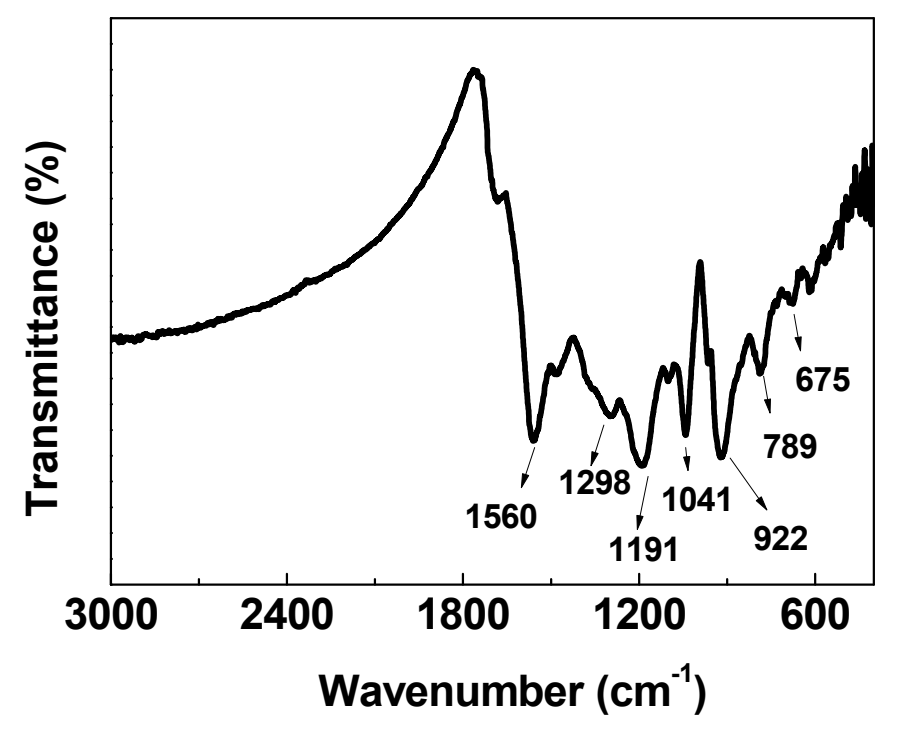

Figure 7. Fourier transform infrared (FTIR) spectrum of polypyrrole.

O1s spectrum can be fitted into three peaks corresponding to quinone $\mathrm{C}=\mathrm{O}(530.6 \pm 0.3 \mathrm{eV})$, $\mathrm{C}-\mathrm{O}-\mathrm{C}(532.9 \pm 0.3 \mathrm{eV})$, and $\mathrm{O}=\mathrm{C}-\mathrm{OH}$ groups $(533.7 \pm 0.3 \mathrm{eV})$. However, the high-resolution $\mathrm{O} 1 \mathrm{~s}$ spectrum of HPC exhibits phenol $\mathrm{C}-\mathrm{OH}(531.8 \pm 0.3 \mathrm{eV}), \mathrm{C}-\mathrm{O}-\mathrm{C}$, and $\mathrm{O}=\mathrm{C}-\mathrm{OH}$ groups (Figure 6e) [19]. Among these oxygen functional groups, only quinone $\mathrm{C}=\mathrm{O}$ in HPGC is able to generate additional faradic pseudocapacitance. Therefore, nickel acetate catalyst can promote the formation of quaternary $\mathrm{N}$ and quinone $\mathrm{C}=\mathrm{O}$ to enhance the conductivity of the material and to generate additional pseudocapacitance. The HPGC can be expected to have higher capacitance and capacity retention than HPC.

In order to evaluate the electrochemical capacitive properties of HPGC, CV curves were performed in $1 \mathrm{M} \mathrm{H}_{2} \mathrm{SO}_{4}$ aqueous electrolyte. Figure 8a displays the $\mathrm{CV}$ curves of HPC and HPGC electrodes at a scan rate of $5 \mathrm{mV} \mathrm{s}^{-1}$. Both HPC and HPGC present similar CV curves which are rectangular-like shape with humps. This is a combined result of electric double layer formation and pseudocapacitive contribution produced by the redox reaction of functional groups, including pyrrolic $\mathrm{N}$, quaternary $\mathrm{N}$, and quinone $\mathrm{C}=\mathrm{O}$. The possible redox reactions show below [22].

$$
\begin{gathered}
>\mathrm{C}=\mathrm{NH}+2 \mathrm{e}^{-}+2 \mathrm{H}^{+} \leftrightarrow>\mathrm{CH}-\mathrm{NH}_{2} \\
>\mathrm{C}-\mathrm{NHOH}+2 \mathrm{e}^{-}+2 \mathrm{H}^{+} \leftrightarrow>\mathrm{C}-\mathrm{NH}_{2}+\mathrm{H}_{2} \mathrm{O} \\
>\mathrm{C}=\mathrm{O}+\mathrm{H}^{+}+\mathrm{e}^{-} \leftrightarrow>\mathrm{C}-\mathrm{OH} \text { (carbonyl, basic) } \\
>\mathrm{C}=\mathrm{O}+\mathrm{e}^{-} \leftrightarrow>\mathrm{C}-\mathrm{O}^{-} \text {(quinone, basic) }
\end{gathered}
$$

Compared with the HPC electrode, the HPGC electrode possesses a larger enclosed area at various scan rates, indicating a higher specific capacitance (Figure $8 \mathrm{a}, \mathrm{b}$ ). Figure $8 \mathrm{c}$, d show the CV curves of two samples at scan rates from 5 to $100 \mathrm{mV} \mathrm{s}^{-1}$ in a voltage of $-0.2-0.8 \mathrm{~V}$. With the increase of scanning rates, the $\mathrm{CV}$ curves of both the HPC and HPGC electrodes maintain a quasi-rectangular shape, implying the good rate performance of HPGC and HPC. The good rate performance can be attributed to the interconnected micro/nano holes that facilitate fast electrolyte transfer. 

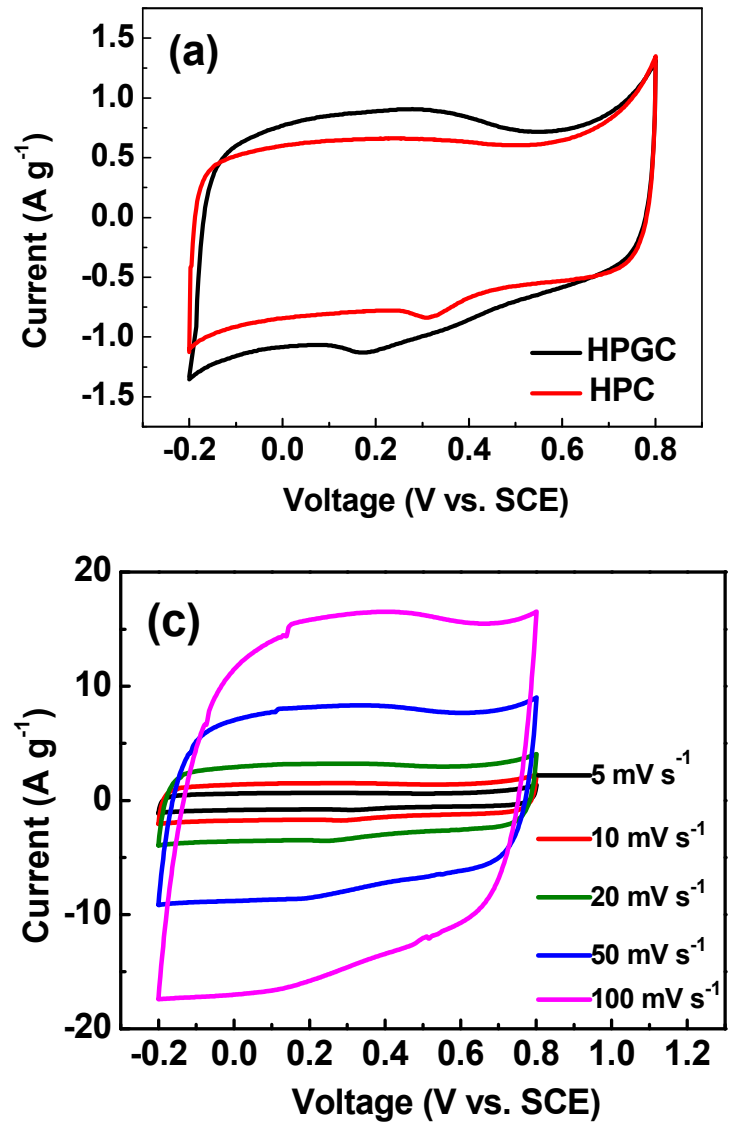
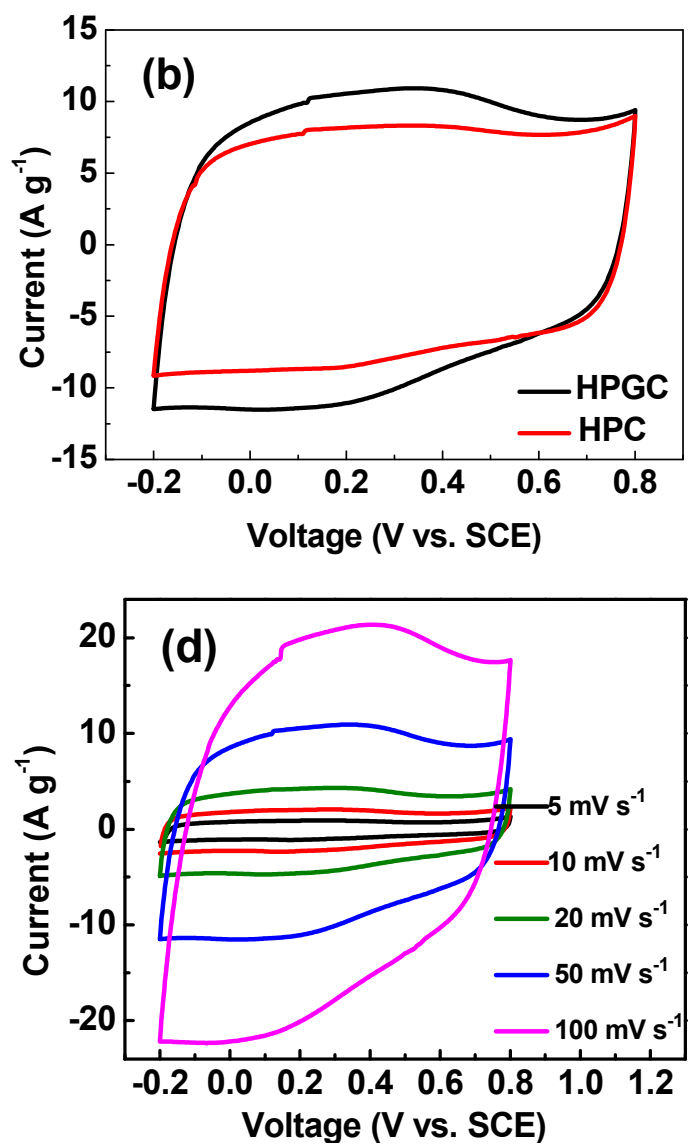

Figure 8. Cyclic voltammetry (CV) curves of HPGC and HPC electrodes at scan rates of $5 \mathrm{mV} \mathrm{s}^{-1}$ (a) and $50 \mathrm{mV} \mathrm{s}^{-1}$ (b); CV curves of HPC (c) and HPGC (d) electrodes at different scan rates from $5 \mathrm{mV} \mathrm{s}^{-1}$ to $100 \mathrm{mV} \mathrm{s}^{-1}$.

Galvanostatic charge-discharge (GCD) measurement is another way to value capacitive properties. Figure 9a,b display the GCD curves for two electrodes at current densities of 0.5 and $5 \mathrm{~A} \mathrm{~g}^{-1}$, respectively. The GCD curves of HPGC and HPC electrodes are not strictly symmetrical. The little deviation from linear property is attributed to the pseudocapacitance effect resulted from functional groups. The deviation in the curve of the HPGC electrode is larger than that of the HPC electrode, suggesting a greater contribution of pseudocapacitance. This is consistent with the result of XPS analysis. Besides, the charge-discharge time of the HPGC electrode is significantly longer than that of the HPC electrode, indicating a higher specific capacitance of HPGC electrode [16]. The result is in good agreement with that obtained from CV curves. Figure 9c,d show the GCD curves of both the HPGC electrode and HPC electrode at various current densities. Both HPGC and HPC display nearly symmetrical curves without obvious voltage drop even at a current density of $50 \mathrm{~A} \mathrm{~g}^{-1}$, suggesting good reversibility for charge storage and transfer. The rate performances of electrodes are reflected in Figure 9e. The HPGC shows much higher specific capacitance values at various current densities, in comparison with the HPC electrode. HPGC electrode delivers capacitance of $336.3 \mathrm{~F} \mathrm{~g}^{-1}$ at $0.5 \mathrm{~A} \mathrm{~g}^{-1}$, which is much higher than that of the HPC electrode $\left(262.2 \mathrm{~F} \mathrm{~g}^{-1}\right)$. The capacitance of the HPGC electrode is $26 \%$ higher than that of the HPC electrode, when the current density is $10 \mathrm{~A} \mathrm{~g}^{-1}$. Furthermore, the as-prepared HPGC shows a higher specific capacitance than most other oxygenand nitrogen-enriched porous carbon materials (Table 2). Even at a high current density of $50 \mathrm{~A} \mathrm{~g}^{-1}$, the specific capacitance of HPGC still reaches as high as $212.2 \mathrm{~F} \mathrm{~g}^{-1}$. However, the HPC electrode only shows a specific capacitance of $110.0 \mathrm{~F} \mathrm{~g}^{-1}$ at $50 \mathrm{~A} \mathrm{~g}^{-1}$. The greatly improved rate capability of HPGC can be ascribed to the graphitization and abundant conjoint pores of HPGC. The limited extent of graphitization may be a major cause of capacity loss with the increase of current density. The HPGC 
electrode also exhibits a higher cycling stability with $97.4 \%$ capacitance retention after 3000 cycles at a current density of $10 \mathrm{~A} \mathrm{~g}^{-1}$ (Figure 9f). For the HPC electrode, the capacitance retention is $94.7 \%$, which is slightly lower than that of HPGC. The remarkable cycling stability of HPGC benefits not only from excellent chemical reversibility of the pseudocapacitive nitrogen- or oxygen-containing functional groups during the charge and discharge process, but also from the enhanced electron conductivity and wettability of the electrolyte towards the electrode surface.
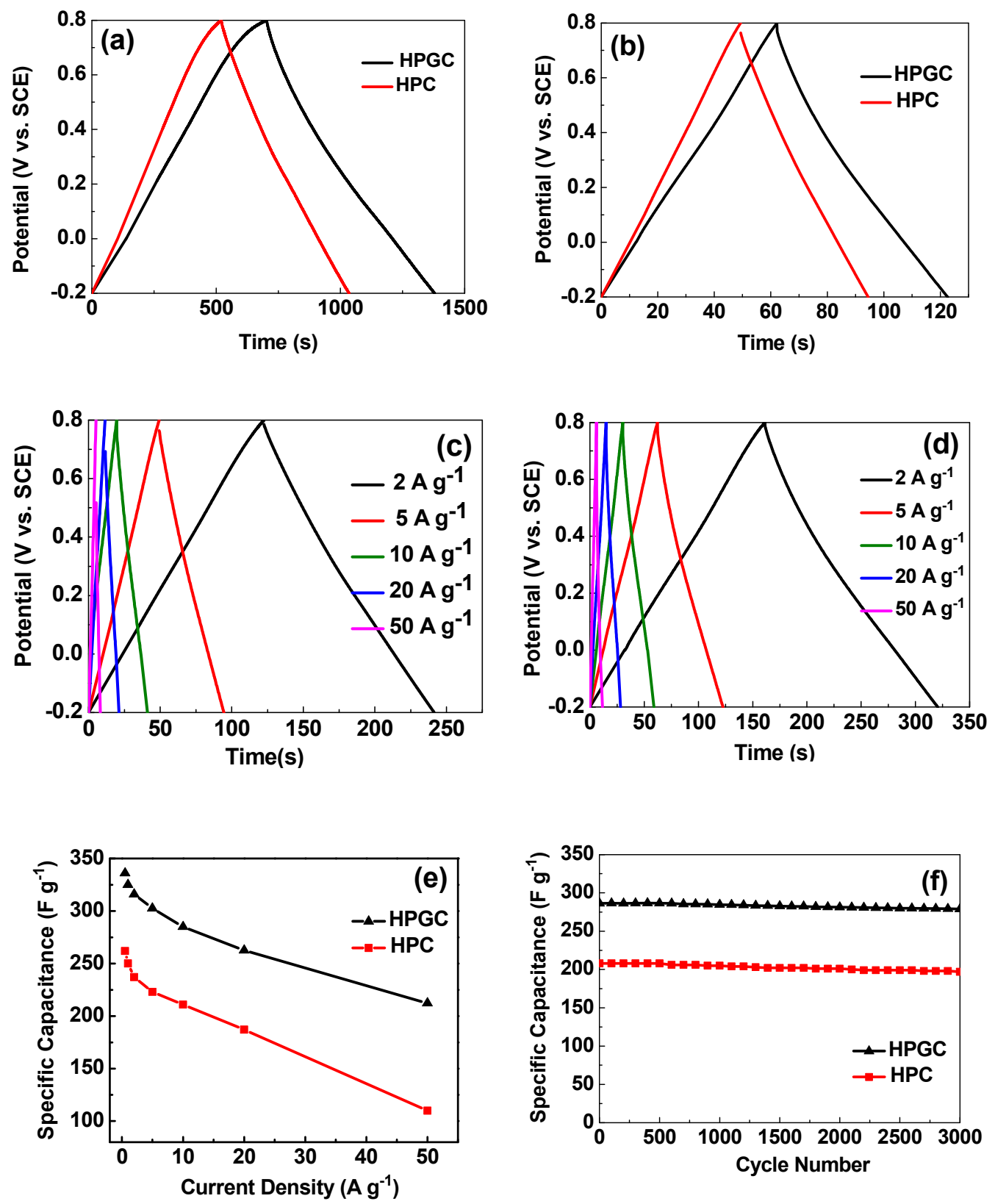

Figure 9. The galvanostatic charge and discharge profiles of the HPGC electrode and HPC electrode at current densities of $0.5 \mathrm{~A} \mathrm{~g}^{-1}$ (a) and $5 \mathrm{~A} \mathrm{~g}^{-1}(\mathbf{b})$; The galvanostatic charge and discharge profiles of the HPC (c) and HPGC (d) electrodes at various current densities. (e) The specific capacitance of the HPC and HPGC electrodes at different current densities; (f) Cycling tests of the HPGC and HPC electrodes at a current density of $10 \mathrm{~A} \mathrm{~g}^{-1}$ for 3000 cycles. 
Table 2. Summary of electrochemical performance for oxygen- and nitrogen-enriched porous carbon electrode materials.

\begin{tabular}{cccc}
\hline Carbon Precursor & SSA $\left.\mathbf{~ m}^{\mathbf{2}} \mathbf{g}^{-\mathbf{1}}\right)$ & Specific Capacitance $\left.\mathbf{( F ~} \mathbf{~ g}^{\mathbf{1}}\right)$ & Reference \\
\hline benzotriazole & 1337.7 & $302.55\left(0.5 \mathrm{~A} \mathrm{~g}^{-1}\right)$ & {$[22]$} \\
kelp & 1000 & $440\left(0.5 \mathrm{~A} \mathrm{~g}^{-1}\right)$ & {$[20]$} \\
potassium humate/manganous nitrate & 1119 & $<225\left(0.5 \mathrm{~A} \mathrm{~g}^{-1}\right)$ & {$[21]$} \\
phenolic resin/polyvinylpyrrolidone & 463 & $228\left(0.2 \mathrm{~A} \mathrm{~g}^{-1}\right)$ & {$[24]$} \\
Polypyrrole & 2489.2 & $336.3\left(0.5 \mathrm{~A} \mathrm{~g}^{-1}\right)$ & This work \\
\hline
\end{tabular}

The Nyquist plots in Figure 10 were employed to confirm the improved kinetics for electron transport in the HPGC supercapacitor. Both HPGC and HPC electrodes display near-vertical slopes at low-frequency, indicating nearly ideal capacitive properties with fast ion diffusion. The low ion migration resistivity at the electrode/electrolyte interface is mainly caused by connected pores structure and good surface wettability by the introduction of heteroatoms. It is noticeable that HPGC has a smaller diameter of the semicircle in the high frequency region, compared with HPC. Hence, the electric resistance of HPGC is lower than that of HPC, owing to the high electronic conductivity of the HPGC electrode. Its superior electrical conductivity results from graphitized carbon and quaternary $\mathrm{N}$, which were produced by carbonization with nickel acetate catalyst. As a result, the increased electrical conductivity of HPGC can lead to improved rate capability and cycling stability.

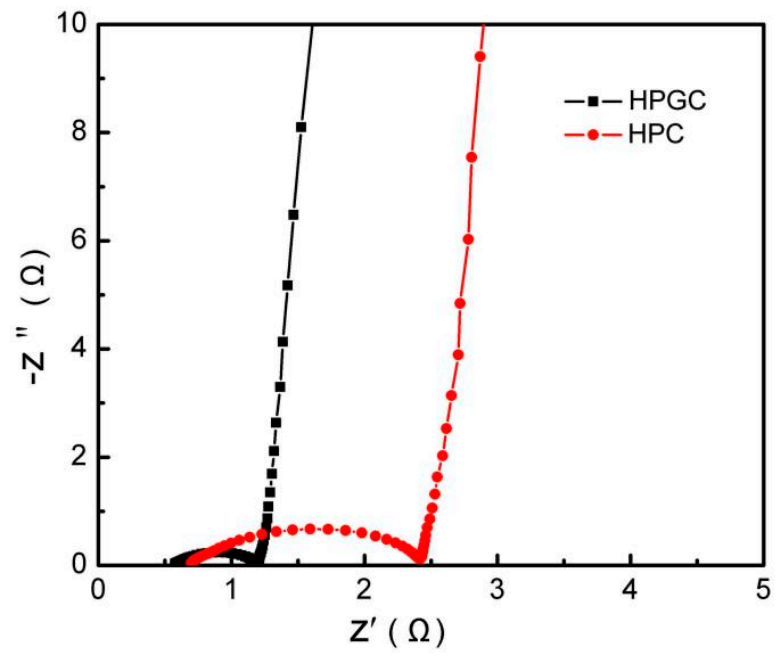

Figure 10. Nyquist plots of HPGC and HPC electrodes.

\section{Conclusions}

In conclusion, we have taken a one-step carbonization-activation-catalytic approach to fabricate novel and functionalized HPGC. It is a significant challenge to derive carbons with both a high graphitization degree and abundant ion-diffusion channels. In our work, Ni catalyst played a significant role in enhancing the electrical conductivity, introducing pseudocapacitance, and promoting ion diffusion. On the one hand, nickel catalyst could promote the formation of graphitized carbon, quaternary $\mathrm{N}$ and quinone $\mathrm{C}=\mathrm{O}$ to enhance the conductivity of the material and to generate additional pseudocapacitance. On the other hand, the retraction and dissolution of $\mathrm{Ni}$ catalyst caused the change of pore size and interconnection of pores in material, obtaining plentiful interfaces for charge accumulation and short paths for ion/electron transport. Therefore, compared with HPC, HPGC shows enhanced energy-storage performances, such as enlarged specific capacity ( $336.3 \mathrm{~F} \mathrm{~g} \mathrm{~g}^{-1}$ at a current density of $\left.0.5 \mathrm{~A} \mathrm{~g}^{-1}\right)$, higher rate capacity (212.2 $\mathrm{F} \mathrm{g}^{-1}$ at a current density of $\left.50 \mathrm{~A} \mathrm{~g}^{-1}\right)$, and more excellent cycling stability ( $97.4 \%$ capacitance retention after 3000 cycles at a current density of $10 \mathrm{~A} \mathrm{~g}^{-1}$ ). Moreover, the as-prepared HPGC shows a higher specific capacitance than most other oxygen- and 
nitrogen-enriched porous carbon materials. HPGC is expected to be a promising electrode material for supercapacitors due to its excellent energy-storage performances and easy mass preparation.

Author Contributions: All authors contributed to the discussion and preparation of the manuscript. Conceptualization, Y.Z.; data curation, H.W. and J.L. (Jinghao Liu); formal analysis, J.L. (Jing Liu); resources, H.P. and K.C.; funding acquisition, G.L.; project administration, Z.Z. All authors have read and agreed to the published version of the manuscript.

Funding: The authors appreciate the financial supports of the Key R\&D project of Shandong Province (2019GGX103034), National Natural Science Foundation of China (No. 21805157, 51672146, 51972187) and the Natural Science Foundation of Shandong Province (ZR2019MEM043, ZR2018BEM011, ZR2019MB037).

Conflicts of Interest: The authors declare no conflict of interest.

\section{References}

1. Wang, F.; Wu, X.; Yuan, X.; Liu, Z.; Zhang, Y.; Fu, L.; Zhu, Y.; Zhou, Q.; Wu, Y.; Huang, W. Latest advances in supercapacitors from new electrode materials to novel device designs. Chem. Soc. Rev. 2017, 46, 6816-6854. [CrossRef] [PubMed]

2. Wang, H.; Liu, J.; Zhang, K.; Peng, H.; Li, G. Meso/microporous nitrogen-containing carbon nanofibers with enhanced electrochemical capacitance performances. Synth. Met. 2015, 203, 149-155. [CrossRef]

3. Shrestha, L.; Shrestha, R.; Maji, S.; Pokharel, B.; Rajbhandari, R.; Shrestha, R.; Pradhananga, R.; Hill, J.; Ariga, K. High surface area nanoporous graphitic carbon materials derived from lapsi seed with enhanced supercapacitance. Nanomaterials 2020, 10, 728. [CrossRef] [PubMed]

4. Li, B.; Dai, F.; Xiao, Q.; Yang, L.; Shen, J.; Zhang, C.; Cai, M. Nitrogen-doped activated carbon for a high energy hybrid supercapacitor. Energy Environ. Sci. 2016, 9, 102-106. [CrossRef]

5. Shao, Y.; El-Kady, M.F.; Wang, L.J.; Zhang, Q.; Li, Y.; Wang, H.; Mousavi, M.F.; Kaner, R.B. Graphene-based materials for flexible supercapacitors. Chem. Soc. Rev. 2015, 44, 3639-3665. [CrossRef]

6. Mao, W.; Yue, W.; Xu, Z.; Chang, S.; Hu, Q.; Pei, F.; Huang, X.; Zhang, J.; Li, D.; Liu, G.; et al. Development of a synergistic activation strategy for the pilot-scale construction of hierarchical porous graphitic carbon for energy storage applications. ACS Nano 2020, 14, 4741-4754. [CrossRef]

7. Jeong, J.; Lee, Y.; Kim, B. Relationship between microstructure and electrochemical properties of 2lignin-derived carbon nanofibers prepared by thermal treatment. Synth. Met. 2020, 260, 116287-116294. [CrossRef]

8. Ma, C.; Xu, J.; Fan, Q.; Shi, J.; Song, Y. Synthesis and electrochemical performance of high surface area hierarchical porous carbon with ultrahigh mesoporosity for high-performance supercapacitors. J. Solid State Electr. 2019, 23, 2153-2163. [CrossRef]

9. Chen, X.; Paul, R.; Dai, L. Carbon-based supercapacitors for efficient energy storage. Natl. Sci. Rev. 2017, 4, 453-489. [CrossRef]

10. Liu, H.; Song, H.; Chen, X.; Zhang, S.; Zhou, J.; Ma, Z. Effects of nitrogen-and oxygen-containing functional groups of activated carbon nanotubes on the electrochemical performance in supercapacitors. J. Power Sources 2015, 285, 303-309. [CrossRef]

11. Li, Y.; Wei, Q.; Wang, R.; Zhao, J.; Quan, Z.; Zhan, T.; Li, D.; Xu, J.; Teng, H.; Hou, W. 3D hierarchical porous nitrogen-doped carbon/ $\mathrm{Ni} @ \mathrm{NiO}$ nanocomposites self-templated by cross-linked polyacrylamide gel for high performance supercapacitor electrode. J. Colloid Interf. Sci. 2020, 570, 286-299. [CrossRef] [PubMed]

12. Hou, L.; Yang, W.; Xu, X.; Deng, B.; Chen, Z.; Wang, S.; Tian, J.; Yang, F.; Li, Y. In-situ activation endows the integrated $\mathrm{Fe}_{3} \mathrm{C} / \mathrm{Fe} @$ nitrogen-doped carbon hybrids with enhanced pseudocapacitance for electrochemical energy storage. Chem. Eng. J. 2019, 375, 122061-122070. [CrossRef]

13. Yun, S.I.; Kim, S.H.; Kim, D.W.; Kim, Y.A.; Kim, B. Facile preparation and capacitive properties of low-cost carbon nanofibers with $\mathrm{ZnO}$ derived from lignin and pitch as supercapacitor electrodes. Carbon 2019, 149, 637-645. [CrossRef]

14. Le, T.; Yoon, H. Strategies for fabricating versatile carbon nanomaterials from polymer precursors. Carbon 2019, 152, 796-817. [CrossRef]

15. Ma, L.; Sun, G.; Ran, J.; Lv, S.; Shen, X.; Tong, H. One-pot template-free strategy toward 3D hierarchical porous nitrogen-doped carbon framework in situ armored homogeneous $\mathrm{NiO}$ nanoparticles for high-performance asymmetric supercapacitors. ACS Appl. Mater. Interfaces 2018, 10, 22278-22290. [CrossRef] 
16. Liu, M.; Zhang, K.; Si, M.; Wang, H.; Chai, L.; Shi, Y. Three-dimensional carbon nanosheets derived from micro-morphologically regulated biomass for ultrahigh-performance supercapacitors. Carbon 2019, 153, 707-716. [CrossRef]

17. Wang, Y.; Liu, R.; Tian, Y.; Sun, Z.; Huang, Z.; Wu, X.; Li, B. Heteroatoms-doped hierarchical porous carbon derived from chitin for flexible all-solid-state symmetric supercapacitors. Chem. Eng. J. 2020, 384, 123263-123272. [CrossRef]

18. Wu, X.; Ding, B.; Zhang, C.; Li, B.; Fan, Z. Self-activation of nitrogen and sulfur dual-doping hierarchical porous carbons for asymmetric supercapacitors with high energy densities. Carbon 2019, 153, 225-233. [CrossRef]

19. Wan, L.; Wei, W.; Xie, M.; Zhang, Y.; Li, X.; Xiao, R.; Chen, J.; Du, C. Nitrogen, sulfur co-doped hierarchically porous carbon from rape pollen as high-performance supercapacitor electrode. Electrochim. Acta 2019, 311, 72-82. [CrossRef]

20. Li, J.; Liu, K.; Gao, X.; Yao, B.; Huo, K.; Cheng, Y.; Cheng, X.; Chen, D.; Wang, B.; Sun, W.; et al. Oxygen-and nitrogen-enriched 3D porous carbon for supercapacitors of high volumetric capacity. ACS Appl. Mater. Interfaces 2015, 7, 24622-24628. [CrossRef]

21. Huang, G.; Geng, Q.; Kang, W.; Liu, Y.; Li, Y.; Xing, B.; Liu, Q.; Zhang, C. Hierarchical porous carbon with optimized mesopore structure and nitrogen doping for supercapacitor electrodes. Micropor. Mesopor. Mater. 2019, 288, 109576-109577. [CrossRef]

22. Chen, H.; Chen, J.; Chen, D.; Wei, H.; Liu, P.; Wei, W.; Lin, H.; Han, S. Nitrogen-and oxygen-rich dual-decorated carbon materials with porosity for high-performance supercapacitors. J. Mater. Sci. 2019, 54, 5625-5640. [CrossRef]

23. To, J.W.F.; Chen, Z.; Yao, H.; He, J.; Kim, K.; Chou, H.; Pan, L.; Wilcox, J.; Cui, Y.; Bao, Z. Ultrahigh surface area three-dimensional porous graphitic carbon from conjugated polymeric molecular framework. ACS Central Sci. 2015, 1, 68-76. [CrossRef] [PubMed]

24. Ma, C.; Cao, E.; Li, J.; Fan, Q.; Wu, L.; Song, Y.; Shi, J. Synthesis of mesoporous ribbon-shaped graphitic carbon nanofibers with superior performance as efficient supercapacitor electrodes. Electrochim. ACTA 2018, 292, 364-373. [CrossRef]

25. Su, P.; Jiang, L.; Zhao, J.; Yan, J.; Li, C.; Yang, Q. Mesoporous graphitic carbon nanodisks fabricated via catalytic carbonization of coordination polymers. Chem. Commun. 2012, 48, 8769-8771. [CrossRef]

26. Wang, K.; Cao, Y.; Wang, X.; Kharel, P.; Gibbons, W.; Luo, B.; Gu, Z.; Fan, Q.; Metzger, L. Nickel catalytic graphitized porous carbon as electrode material for high performance supercapacitors. Energy 2016, 101, 9-15. [CrossRef]

27. Lu, Q.; Weng, Z. Synthesis and characterization of polypyrrole nanoparticles via unstirred polymerization. ACTA Polym. Sin. 2009, 6, 513-519. [CrossRef]

28. Song, M.; Kim, Y.; Kim, B.; Kim, J.; Char, K.; Rhee, H. Synthesis and characterization of soluble polypyrrole doped with alkylbenzenesulfonic acids. Synth. Met. 2004, 141, 315-319. [CrossRef]

29. Han, J.; Li, Q.; Peng, C.; Shu, N.; Pan, F.; Wang, J.; Zhu, Y. Increasing S dopant and specific surface area of N/S-codoped porous carbon by in-situ polymerization of PEDOT into biomass precursor for high performance supercapacitor. Appl. Surf. Sci. 2020, 502, 144191. [CrossRef]

30. Chameh, B.; Moradi, M.; Kaveian, S. Synthesis of hybrid ZIF-d erived binary ZnS/CoS composite as high areal-capacitance supercapacitor. Synth. Met. 2020, 260, 116262. [CrossRef]

31. Wang, Q.; Xu, B.; Xu, C.; Wang, Y.; Zhang, Y.; Wu, J.; Fan, G. Ultrasmall Rh nanoparticles decorated on carbon nanotubes with encapsulated Ni nanoparticles as excellent and $\mathrm{pH}$-universal electrocatalysts for hydrogen evolution reaction. Appl. Surf. Sci. 2019, 495, 143569-143570. [CrossRef]

32. Zhu, Q.; Xia, W.; Zheng, L.; Zou, R.; Liu, Z.; Xu, Q. Atomically dispersed Fe/N-doped hierarchical carbon architectures derived from a metal-organic framework composite for extremely efficient electrocatalysis. ACS Energy Lett. 2017, 2, 504-511. [CrossRef]

33. Saravanan, C.; Shekhar, R.; Palaniappan, S. Synthesis of polypyrrole using benzoyl peroxide as a novel oxidizing agent. Macromol. Chem. Phys. 2006, 207, 342-348. [CrossRef]

(C) 2020 by the authors. Licensee MDPI, Basel, Switzerland. This article is an open access article distributed under the terms and conditions of the Creative Commons Attribution (CC BY) license (http://creativecommons.org/licenses/by/4.0/). 